\title{
A New Application on Gaussian Mixture Modeling in Object Detection
}

\author{
Dawei Qiu, a , Jing Liu, ${ }^{*}$, b and Hui Cao ${ }^{1}$ \\ ${ }^{1}$ College of Science and Engineer, Shandong University of Traditional Chinese Medicine, Jinan, \\ Shandong Province, China \\ adwqiu@foxmail.com, ${ }^{b}$ liuj_jn@163.com
}

\begin{abstract}
Keywords: Object Detection, Gaussian Mixture Model, Maximum Likelihood.
Abstract. For background modeling, the conventional Gaussian Mixture Model (GMM) is a popular approach. However, because of the inappropriate parameters updating method, GMM often suffers from a problem that it cannot classify a pixel into background or foreground correctly for longtime. In the paper, we proposed a new parameters updating method for GMM, and built background model for every pixel and global foreground model for the entire image. We presented an improved object detection and tracking scheme based on the proposed approach. The experimental results show the proposed GMM parameters updating method, together with the object detection and tracking framework, give better performance than the conventional Gaussian Mixture Modeling algorithm.
\end{abstract}

\section{Introduction}

The detection of regions of interest is the important step in many computer vision applications, such as visual surveillance, event detection, image understanding etc. A general object detection technique is desired, but it is very difficult to detect objects even unknown objects under complex environments. So many computer vision applications assume stable environments or few changes in scenes, in which the background model is trained and foreground regions are obtained via the difference between current frame and the background model, the task of object detection implementation is straightforward. The process is called background subtraction.

In recent years, many researchers have proposed various background modeling and subtraction algorithms. Background modeling methods consists of pixels-based and blocks-based [1]. Background modeling based on pixels builds models according to every pixel's distribution information in time fields. In [2] each pixel is modeled by a Gaussian model. From that, Gaussian models are more and more used in background subtraction. Friedman et al. N. Friedman and S. Russell [3] proposed Mixture of Gaussians in traffic surveillance, each pixel consists of three Gaussian components corresponding to car, road and shadow respectively. Stauffer et al. C. Stauffer [4] proposed more general Gaussian Mixture Model (GMM) method. The adaptive background subtraction approach models each pixel as a mixture of Gaussians and uses on-line approximation to update the model. Elgammal et al. [5] proposed a nonparametric background modeling method, which utilizes general nonparametric kernel density estimation techniques for building statistical representations of the foreground and the background without any assumptions about the underlying distributions. Y. Ren et al. [6] proposed a new background subtraction approach, which can deal with non-stationary scenes. The method classified a pixel in the current frame into foreground or background based on the temporally and spatially modeled distribution of each background pixel. After that, object detection with nonstationary background is referred to by more and more researchers. In [7], the authors proposed a new Gaussian matching criteria and reduced the calculation burden.

Background modeling based on blocks segments the frame into blocks, background model is then built using features of each block. Compared with pixel-based method, block-based method can utilize more spatial distribution information and is insensitive to local changes in the scene. Matsuyama et al. T. Matsuyama et al. [8] used normalized vector distance measure correlation between different image blocks. In [9], LBP (Local Binary Pattern) histogram in each pixel's neighborhood is used to represent the background model. The drawback of these algorithms is that the obtained moving objects regions

\footnotetext{
* Correspondence author
} 
are coarse and are not suitable for precise object segmentation. In [10], the authors proposed an efficient hierarchical method for background subtraction, the method combines pixel-based and block-based approaches into a single framework, and these two approaches are complementary to each other.

In this paper, we propose a new object detection algorithm based on an improved Gaussian Mixture Model. The algorithm uses Maximum Likelihood (ML) approach to update the GMM parameters and builds background and foreground models with GMM respectively. The new pixel is classified as background or foreground according to these two models. Experimental results show that the proposed algorithm can improved the precision and performance than the conventional GMM approach.

The rest of the paper is organized as follows. Section 2 describes the conventional GMM algorithm, the proposed algorithm is detailed in Section 3, and the experimental results are presented in Section 4. Finally, brief conclusions are given in Section 5.

\section{Proposed Object Detection Algorithm}

In this paper, we propose a new object detection algorithm based on an improved Gaussian Mixture Model. The algorithm uses Maximum Likelihood (ML) approach to update the parameters of the Gaussian Mixture Models. Then every pixel is classified as background or foreground, the algorithm maintain background and foreground model, respectively. The flow chart of the entire algorithm is shown in Fig. 1:

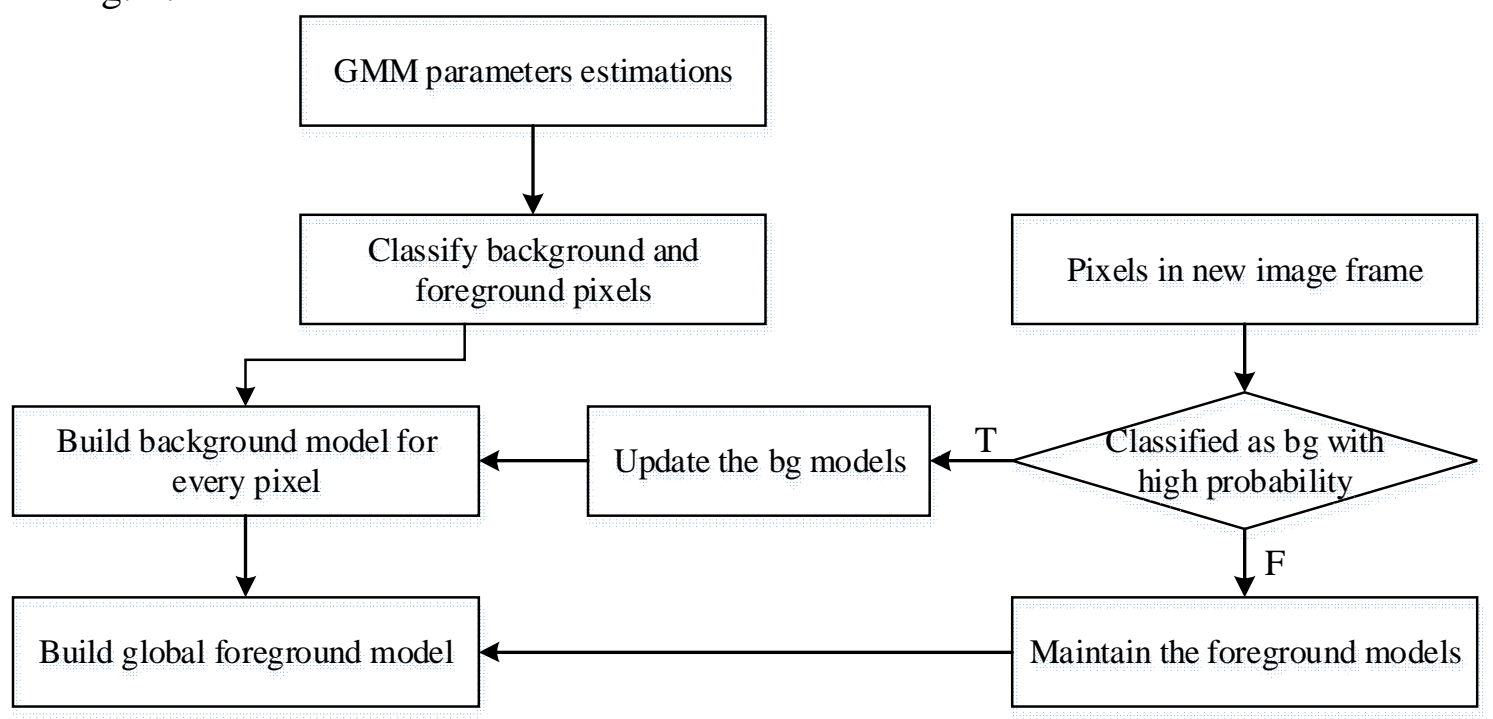

Fig. 1 The flow chart of the proposed algorithm

For every pixel, the observation sequence is $X_{1}, X_{2}, \ldots, X_{t}$, the maximum likelihood function of GMM is:

$$
\sum_{j=1}^{t} \log \left\{\sum_{k=1}^{K} \gamma_{k} N\left(X_{j, t} \mid \mu_{k}, \Sigma_{k}\right)\right\}
$$

The above equation is hard to optimize, so we adopt the following two steps:

Step 1. Estimate the probability of the pixel belongs to each Gaussian component, it can be computed as:

$$
\gamma_{k}=\frac{\pi_{k} N\left(X_{t} \mid \mu_{k}, \Sigma_{k}\right)}{\sum_{j=1}^{K} \pi_{j} N\left(X_{t} \mid \mu_{j}, \Sigma_{j}\right)}
$$

Step 2. Estimate the parameters of each Gaussian component: 


$$
\begin{aligned}
& \mu_{k}=\frac{1}{N_{k}} \sum_{j=1}^{t} \gamma_{k} X_{j} \\
& \Sigma_{k}=\frac{1}{N_{k}} \sum_{j=1}^{t} \gamma_{k}\left(X_{j}-\mu_{k}\right)\left(X_{j}-\mu_{k}\right)^{T}
\end{aligned}
$$

The proposed algorithm is as follows.

Step 1. Build background and global foreground models with the first image sequences according to Eq. 4 and Eq. 10-12.

Step 2. For new observations, classify it as background pixel if the pixel matches its background models with predefined high probability, and update the corresponding Gaussian component parameters and weights of other components, go to step 5, else go to step 3.

Step 3. If the pixel matches foreground model with high probability, update the foreground model, go to step 5, else go to step 4.

Step 4. If the pixel does not match the foreground model with high probability, classify it as background model, and build new Gaussian component to add it to its background model or replace the Gaussian component with lowest weight.

Step 5. For new image frames, repeat Steps 2-4 until end.

\section{Experimental Results}

In order to evaluate the performance of the proposed algorithm, we run our algorithm and the conventional GMM method on several widely used datasets. The algorithms are implemented using MATLAB 2013a on a Pentium IV, 2.40GHz, 4G RAM computer. The datasets come from PETS2001 [11], the resolution of the images is $768 \times 576$. The experimental results are shown in Fig. 2 , the left columns are the results of the conventional GMM, and the right columns below are the results of the proposed algorithm.
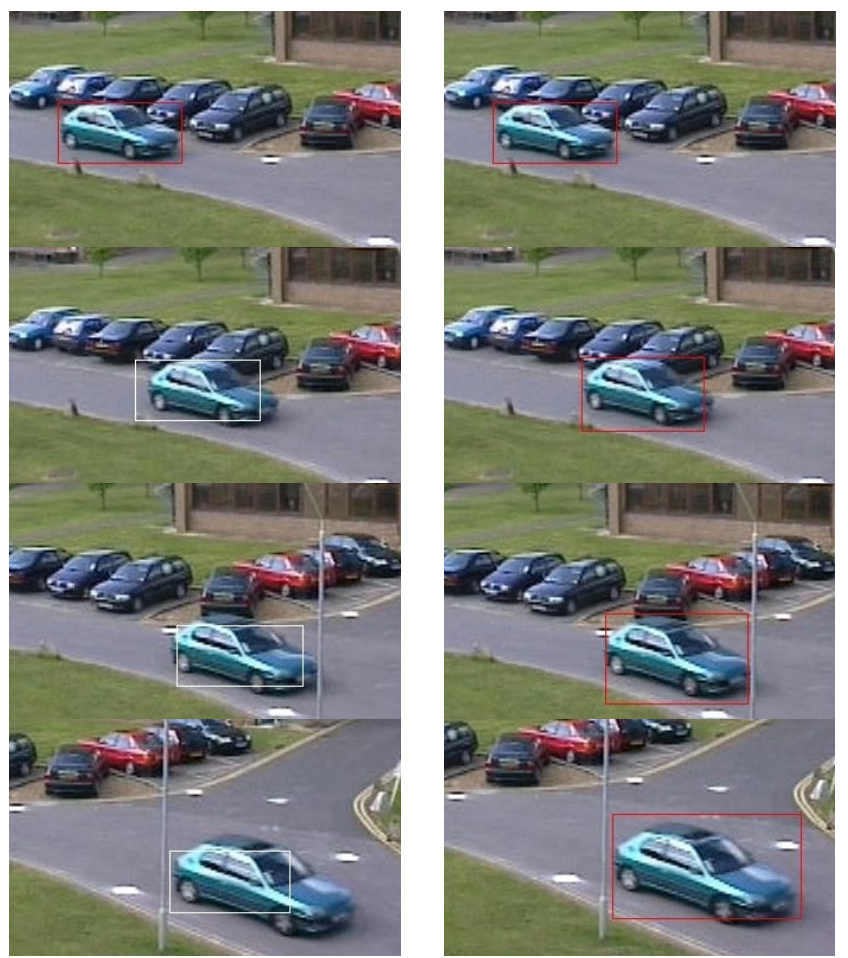

Fig. 2 tracking results of the conventional GMM and the proposed algorithm

From the experiment, we can see that for the inappropriate parameters updating method of the conventional GMM approach, the algorithm cannot track objects longtime and precisely. Because accurate parameters updating method, our proposed algorithm can track object precisely. 
Due to limited space, we show an experiment here, and several other experiments also demonstrate the efficiency and precision of our algorithm.

\section{Conclusion}

In this work, we adopt a new parameters updating method for Gaussian Mixture Model, and build global foreground model to detect objects in image sequences. The experimental results show the effectiveness of the proposed approach. But the method need more computation resources to fulfil the parameters updating process than the conventional GMM. In the future, we will make improvements to speed up the algorithm without loss of precision.

\section{Acknowledgements}

This work was financially supported by the Science and Research Development Projects of Shangdong Province (J15LL04) and the National Natural Science Foundation (NO. 81473708).

\section{References}

[1] Yongzhong Wang, Yan Liang \& Quan Pan, Yongmei Cheng \& Chunhui Zhao. Spatiotemporal Background Modelling Based on Adaptive Mixture of Gaussians. ACTA AUTOMATICA SINICA, 35(2009), 371-378.

[2] C. R. Wren, A. Azarbayejani, T. Darrel \& A. P. Pentland. Pfinder: real-time tracking of the human body. IEEE Transactions on Pattern Analysis and Machine Intelligence, 19(1997), 780-785.

[3] N. Friedman \& S. Russell. Image segmentation in video sequences: a probabilistic approach. USA: Morgan Kaufmann 1997: Proceedings of the 13th Conference on Uncertainty in Artificial Intelligence. Providence. pp. 175-181.

[4] C. Stauffer \& W. Eric L. Grimson. Learning Patterns of Activity Using Real-Time Tracking. IEEE Transactions on Pattern Analysis and Machine Intelligence, 22(2000), 747-757.

[5] A. Elgammal, R. Duraiswami, D. Harwood \& L. S. Davis. Background and Foreground Modeling Using Nonparametric Kernel Density Estimation for Visual Surveillance. Proceedings of the IEEE, 90(2002), 1151-1163.

[6] Y. Ren, C. Chua \& Y. Ho. Motion detection with non-stationary background. Machine Vision and Applications, 13(2003), 332-343.

[7] Xiangfeng Bai, Aihua Li, Xilai Li \& Renbing Li. A Novel Background Gaussian Mixture Model. Journal of Image and Graphics, 16(2011), 983-988.

[8] T. Matsuyama, T. Ohya \& H. Habe. Background subtraction for non-stationary scenes. Taipei, Taiwan: Proceedings of the 4th Asian Conference on Computer Vision. University Trier Press. (2000) 114-116.

[9] M. Heikkila \& M. Pietikainen. A Texture-Based Method for Modelling the Background and Detecting Moving Objects. IEEE Transactions on Pattern Analysis and Machine Intelligence, 28(2006), 657-662.

[10] Y. Chen, C. Chen, C. Huang \& Y. Hung. Efficient hierarchical method for background subtraction. Pattern Recognition, 40 (2007), 2706-2715.

[11] Pets2001. [Online]. Available from: http://www.anc.ed.ac.uk/demos/tracker/pets2001.html. 